\title{
miR-363 inhibits the growth, migration and invasion of hepatocellular carcinoma cells by regulating E2F3
}

\author{
JUNFENG YE, WEI ZHANG, SONGYANG LIU, YAHUI LIU and KAI LIU
}

Department of Hepatopancreatobiliary Surgery, The First Hospital of Jilin University, Changchun, Jilin 130021, P.R. China

Received November 18, 2016; Accepted June 1, 2017

DOI: $10.3892 /$ or.2017.6018

\begin{abstract}
A growing body of evidence suggests that microRNA-363 (miR-363) plays crucial roles in tumor progression, development and metastasis, and confer resistance to chemotherapeutic drugs in several types of cancers. However, the biological function and underlying molecular mechanism of miR-363 in hepatocellular carcinoma (HCC) have not been fully elucidated. In the present study, we investigated the biological function and mechanism of miR-363 in the regulation of $\mathrm{HCC}$ progression. We found that miR-363 was downregulated in HCC cell lines and tissues, and a low expression level of miR-363 was associated with tumor differentiation, TNM stage and lymph node metastasis. Forced overexpression of miR-363 significantly suppressed HCC cell proliferation, migration, invasion and decreased epithelial-mesenchymal transition (EMT) in vitro, as well as inhibited tumor growth in vivo. Analysis of the underlying mechanisms revealed that miR-363 regulated E2F transcription factor 3 (E2F3) expression by directly targeting its $3^{\prime}$ untranslated region. E2F3 overexpression partially attenuated the tumor-suppressive effects of miR-363 in HCC cells. In addition, E2F3 expression was upregulated in the $\mathrm{HCC}$ tissues, and was negatively correlated with the level of miR-363 in human HCC tissues. Taken together, these results revealed that miR-363 is involved in HCC growth and invasion and functions as a tumor suppressor by negatively regulating E2F3.
\end{abstract}

\section{Introduction}

Hepatocellular carcinoma (HCC) is one of the most common malignant tumors in China, and an increasing incidence has been reported worldwide (1). Although multimodal treatments such as surgical resection, liver transplantation, chemotherapy and radiotherapy or a combination of these options have been

Correspondence to: Dr Kai Liu, Department of Hepatopancreatobiliary Surgery, The First Hospital of Jilin University, Changchun, Jilin 130021, P.R. China

E-mail: liukai514@126.com

Key words: hepatocellular carcinoma, miR-363, E2F3, migration, invasion performed in recent years, the long-term survival of HCC patients remains extremely poor due to a high incidence of recurrence and metastasis $(2,3)$. Therefore, understanding of the underlying molecular mechanisms leading to the initiation and metastasis of HCC are required for the development of more effective therapeutic strategies.

MicroRNAs (miRNAs) are a class of short, endogenous, non-coding RNAs known to negatively regulate the expression of protein-coding genes by inhibiting translation or inducing messenger RNA (mRNA) degradation by binding to the 3' untranslated regions (3'UTRs) of target genes (4,5). Since miRNAs are able to regulate multiple targets (6), miRNAs are involved in the regulation of various biological processes, including proliferation, apoptosis, metastasis and angiogenesis (7). Alterations in miRNA expression have been reported to play essential roles in tumorigenesis and tumor progression processes, and function as oncogenes or tumor suppressors (8). Accumulating evidence has suggested that alterations in miRNA expression contribute to HCC development and progression, and can serve as diagnostic and prognostic markers, and therapeutic agents for $\operatorname{HCC}(9,10)$.

The aberrant expression of microRNA-363 (miR-363) and its role in tumor pathogenesis and progression have already been reported in gallbladder (11), colorectal (12), gastric (13), and head and neck (14), as well as in breast cancer (15). In HCC, miR-363 expression was found to be downregulated and inhibited cell proliferation by downregulating the expression of S1PR (16), and decreased the cisplatin resistance of HCC cells, partly by targeting Mcl-1 (17). However, the detailed biological role, particularly with respect to metastasis in HCC, has not been fully elucidated. In the present study, we found that miR-363 was decreased in HCC tissues and cell lines. In addition, a low expression level of miR-363 was associated with tumor differentiation, tumor-node-metastasis (TNM) stage and lymph node metastasis. We found that miR-363 overexpression significantly inhibited HCC cell proliferation, migration and invasion in vitro and suppressed tumor growth in vivo by targeting E2F transcription factor 3 (E2F3). These results suggest that miR-363 may be a novel target for HCC.

\section{Materials and methods}

Patients and clinical tissue specimens. Forty pairs of primary HCC and adjacent non-tumor tissues were obtained from patients undergoing surgery at the Department of 
Hepatopancreatobiliary Surgery, The First Hospital, Jilin University (Changchun, China). The collection and use of patient samples was approved by the Ethics Committee of the First Hospital of Jilin University, and written informed consent was obtained from all patients whose biological samples were used in the study. All samples were immediately snap-frozen in liquid nitrogen and stored at $-80^{\circ} \mathrm{C}$ until use.

Cell culture and transfection. The $4 \mathrm{HCC}$ cell lines (SMMC-7721, Hep3B, HepG2 and Huh-7) as well as the normal human hepatocyte cell line HL-7702, were purchased from the Institute of Biochemistry and Cell Biology, Chinese Academy of Sciences (Shanghai, China). All cell lines were maintained in high glucose Dulbecco's modified Eagle's medium (DMEM) supplemented with $10 \%$ fetal bovine serum (FBS) (both from Gibco, Gaithersburg, MD, USA) at $37^{\circ} \mathrm{C}$ in a humidified chamber supplemented with $5 \% \mathrm{CO}_{2}$.

The miR-363 mimic and the appropriate negative control oligonucleotide (miR-NC) were purchased from RiboBio (Guangzhou, China). The E2F3 overexpression vector was amplified and inserted into the pcDNA3.0 vector (Invitrogen Life Technologies, Grand Island, NY, USA) at the BamHI/EcoRI restriction sites. Transfection was performed using Lipofectamine 2000 (Invitrogen) in accordance with the manufacturer's protocol.

RNA isolation and $q R T-P C R$. Total RNA containing miRNA and mRNA was extracted from tissue samples and cells using TRIzol ${ }^{\circledR}$ reagent (Invitrogen) according to the manufacturer's instructions. RNA was reverse transcribed to cDNA using Prime Script First Strand cDNA Synthesis kit (Takara, Daliạn, China) following the manufacturer's protocol. The cDNAs were subjected to qRT-PCR using SYBR Premix Ex Taq (Takara) under ABI 7900 Fast System (Applied Biosystems, Foster City, CA, USA) to detect miR-363 and E2F3 mRNA. miR-363 and U6 primers were purchased from RiboBio. The primers for E2F3 and GAPDH used in the present study were as previously described (18). U6 and GAPDH were used as internal controls to detect miR-363 and E2F3 mRNA, respectively. Relative expression of miR-363 and E2F5 was determined using the $2^{-\Delta \Delta \mathrm{Ct}}$ method.

Cell proliferation and colony formation assays. Cell proliferation was assessed using Cell Counting Kit-8 (CCK-8) assay (Dojindo Laboratories, Kumamoto, Japan) according to the manufacturer's instructions. In brief, $5 \times 10^{3}$ transfected cells/well were seeded in 96-well plates and cultured for 24-72 h. At the indicated time, an amount of $20 \mu \mathrm{l}$ of CCK-8 solution was added to each well and incubation was carried out for $4 \mathrm{~h}$. Absorbance at $450 \mathrm{~nm}$ was measured using an enzymelinked immunosorbent assay reader (Thermo Labsystems, Helsinki, Finland).

Colony formation ability was determined using plating at 500 transfected cells/well into 6-well plates and culturing for 14 days. Cells were fixed with methanol and stained with $0.1 \%$ crystal violet for $15 \mathrm{~min}$. Stained colonies were imaged and counted by a light microscope (Olympus, Tokyo, Japan).

Cell migration and invasion assays. Cell migration was measured using a wound healing assay. In brief, transfected cells were cultured in 6 -well plates $\left(5 \times 10^{4}\right.$ cells/well) and grown to confluency. Subsequently, an artificial homogenous wound was scratched into the monolayer using a sterile plastic micropipette tip, and then the cells were cultured under standard conditions for $24 \mathrm{~h}$. Following several washes, the cultured cells were fixed and observed using an inverted microscope (Olympus).

The invasion assay was performed using Transwell insert chambers (Corning Inc., Corning, NY, USA). For the Transwell invasion assay, $1 \times 10^{5}$ transfected cells were seeded into each well of the upper chamber of Matrigel-coated inserts in serum-free medium. In the lower chamber, DMEM with $20 \%$ FBS was added to serve as a chemoattractant. After incubating for $24 \mathrm{~h}$ at $37^{\circ} \mathrm{C}$ with $5 \% \mathrm{CO}_{2}$, the non-invading cells were gently removed with a cotton swab, whereas cells that had invaded to the lower surface of the filter were fixed in $70 \%$ ethanol for $30 \mathrm{~min}$ and stained with $2 \%$ crystal violet for $10 \mathrm{~min}$. Invaded cells were photographed and quantified by counting them randomly in 5 fields by a light microscope (Olympus).

Dual-luciferase reporter assay. The 3'UTR of E2F3 containing the potential binding sites of miR-363 (position 272-278) was amplified by PCR using human liver cDNA, and inserting into the psiCHECK2 vector (Promega, Wisconsin, WI, USA) within the XhoI/NotI restriction sites. A mutant 3'UTR of E2F3 was constructed using QuikChange XL Site-Directed Mutagenesis kit (Agilent Technologies, Santa Clara, CA, USA), and cloned into the psiCHECK2 vector. For the luciferase reporter assay, HepG2 cells were seeded in a 96-well plate. When reaching $70 \%$ confluency, the cells were cotransfected with miR-363 mimic/miR-NC and wild/mutant-type 3'UTR of E2F3. Luciferase activities were determined $48 \mathrm{~h}$ after transfection using the Dual-Luciferase Reporter Assay System (Promega).

Western blot analysis and antibodies. Total protein extraction, SDS-PAGE and western blot analyses were performed as previously described (19). The primary antibodies against E2F3, GAPDH, E-cadherin, N-cadherin and vimentin were purchased from Cell Signaling Technology Inc. (Danvers, MA, USA). GAPDH was used as an internal control for all experiments.

Xenograft mouse model. For in vivo tumorigenesis assays, $2 \times 10^{6}$ HepG2 cells carrying either miR-363 or miR-NC were subcutaneously injected into the flanks of female BALB/c nude mice (6-7 weeks of age) (Laboratory Animal Center of Jilin University). Tumor volume was determined by measuring tumor length (L) and width (W) every 7 days using the formula: Tumor volume $=0.5 \times\left(\mathrm{L} \mathrm{x} \mathrm{W}^{2}\right)$. At 35 days after injection, animals were sacrificed and subcutaneous tumors were stripped and weighed. All animal handling and research protocols were approved by the Animal Care and Use Ethics Committee of Jilin University.

Statistical analysis. In all experiments, the data are expressed as the mean \pm standard error of the mean (SEM) from at least 3 independent experiments. The differences between groups were analyzed using Student's t-test when only 2 groups were compared, or using a one-way ANOVA when $>2$ groups were compared. Correlations between miR-363 expression with E2F3 were evaluated by the Pearson's correlation analysis. A 

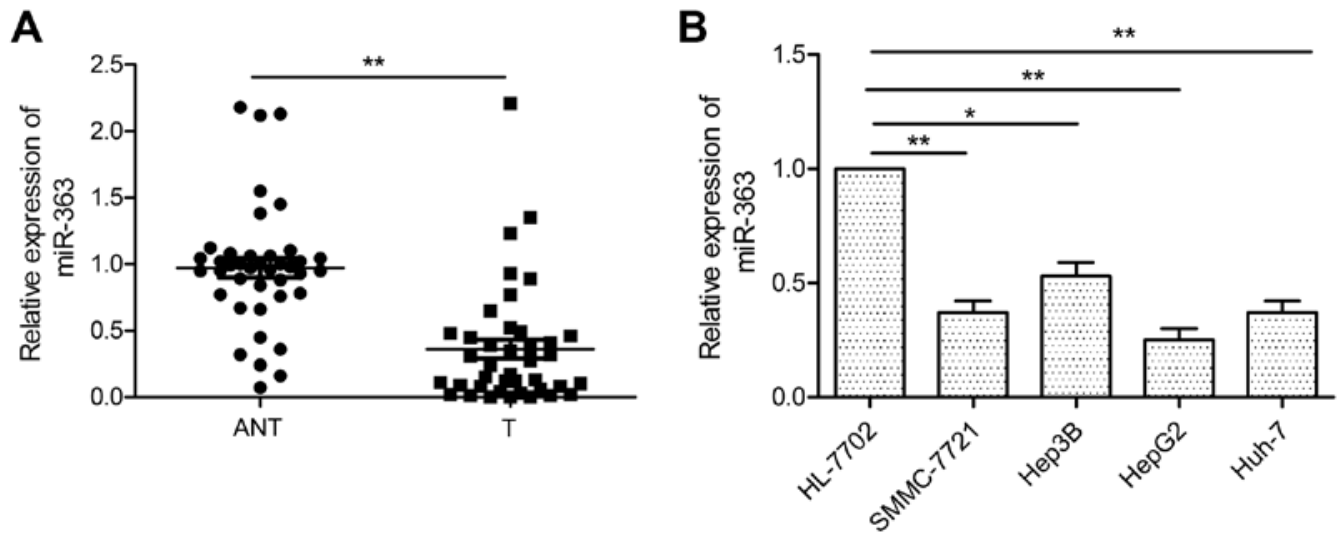

Figure 1. miR-363 expression is decreased in HCC tissues and cell lines. (A) The expression of miR-363 in 40 paired HCC (T) and adjacent non-tumor tissues (ANT) was determined using qRT-PCR. (B) The expression of miR-363 was determined in 4 HCC cell lines (SMMC-7721, Hep3B, HepG2 and Huh-7) and a normal human hepatocyte cell line, HL-7702 by qRT-PCR; ${ }^{*} \mathrm{P}<0.05,{ }^{* *} \mathrm{P}<0.01$.

value of $\mathrm{P}<0.05$ was considered as an indication of statistical significance. All analyses were performed using SPSS 19.0 software (SPSS, Inc., Chicago, IL, USA).

\section{Results}

miR-363 is decreased in HCC tissues and cell lines. We examined the expression of miR-363 in 40 paired $\mathrm{HCC}$ and corresponding adjacent non-tumor tissues by qRT-PCR. We found that miR-363 was significantly decreased in the HCC tissues (Fig. 1A). To assess the association of miR-363 expression with HCC prognosis, patients were divided into 2 groups using the mean miR-363 expression (0.33) as cut-off: one with relative high miR-363 expressions $(>0.33)$, and another with relative low miR-363 expressions $(<0.33)$. We found that miR-363 expression was significantly associated with TNM stage, tumor differentiation and lymph node metastasis (Table I). We next examined the expression of miR-363 in a series of HCC cell lines. We found that the expression of miR-363 in 4 human HCC cell lines (SMMC-7721, Hep3B, HepG2 and Huh-7) was significantly downregulated compared with that noted in the normal human hepatocyte cell line, HL-7702 (Fig. 1B). These data suggest that miR-363 is involved in the carcinogenesis of HCC.

miR-363 inhibits HCC growth in vitro and in vivo. To assess the functional significance of miR-363 in HCC growth, HepG2 cells with low expression of miR-363 were transfected with the miR-363 mimic (miR-363) or miR-NC, and then cell proliferation and colony formation ability were determined. qRT-PCR results confirmed that miR-363 was upregulated in the HepG2 cells transfected with the miR-363 mimic as compared to cells transfected with miR-NC (Fig. 2A). CCK-8 assay revealed that overexpession of miR-363 significantly suppressed proliferation of the HepG2 cells (Fig. 2B). Consistent with this result, overexpression of miR-363 also significantly suppressed colony formation of the HepG2 cells (Fig. 2C). To further confirm whether miR-363 suppresses tumor growth in vivo, we created tumor xenograft mouse models. Consistently, miR-363 significantly inhibited tumor growth in vivo (Fig. 2D). After 35 days of injection, the mice were sacrificed, and tumor tissues were
Table I. Association of miR-363 expression with clinicopathological factors of the $40 \mathrm{HCC}$ patients.

\begin{tabular}{|c|c|c|c|c|}
\hline & & miR-363 & xpression & \\
\hline Variables & $\begin{array}{l}\text { No. of } \\
\text { cases }\end{array}$ & $\begin{array}{l}\text { Low } \\
\mathrm{n}(\%)\end{array}$ & $\begin{array}{l}\text { High } \\
\text { n }(\%)\end{array}$ & P-value \\
\hline Age (years) & & & & $>0.05$ \\
\hline$<55$ & 19 & $10(52.6)$ & $9(47.4)$ & \\
\hline$\geq 50$ & 21 & 13 (61.9) & $8(38.1)$ & \\
\hline Sex & & & & $>0.05$ \\
\hline Male & 23 & $14(60.8)$ & $9(39.2)$ & \\
\hline Female & 17 & $9(52.9)$ & $8(47.1)$ & \\
\hline TNM stage & & & & $<0.01$ \\
\hline I-II & 29 & $12(41.4)$ & $17(58.6)$ & \\
\hline III-IV & 11 & $11(100)$ & $0(0)$ & \\
\hline Differentiation & & & & $<0.01$ \\
\hline Well/moderate & 30 & $14(46.7)$ & $16(53.3)$ & \\
\hline Poor & 10 & $9(90.0)$ & $1(10.0)$ & \\
\hline $\begin{array}{l}\text { Lymph node } \\
\text { metastasis }\end{array}$ & & & & $<0.01$ \\
\hline No & 31 & $14(45.2)$ & $17(54.8)$ & \\
\hline Yes & 9 & $9(100)$ & $0(0)$ & \\
\hline
\end{tabular}

miR-363, microRNA-363; HCC, hepatocellular carcinoma; TNM, tumor-node-metastasis.

stripped. We found that the size of the subcutaneous tumors derived from the miR-363-expressing HepG2 cells were markedly smaller than the size of the tumors derived from the miR-NC-transfected cells (Fig. 2E). Taken together, our data support a growth inhibitory activity of miR-363 in HCC in vitro and in vivo.

miR-363 inhibits HCC migration and invasion, and ETM. We next studied the effects of miR-363 on cell migration and 
A

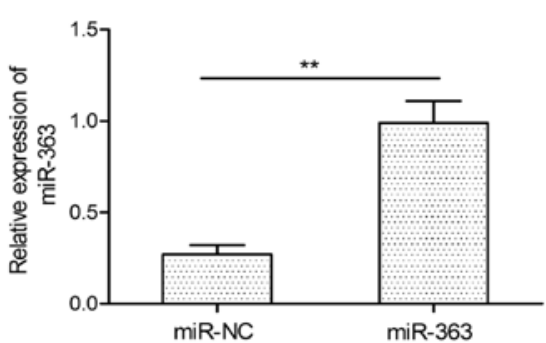

C
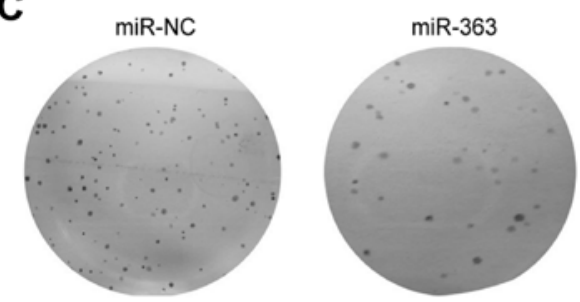

D

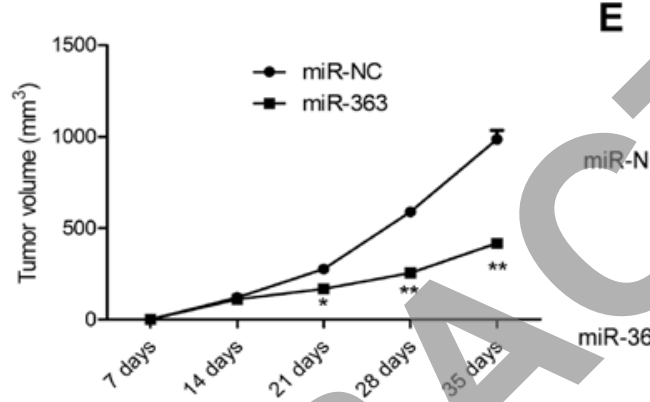

B
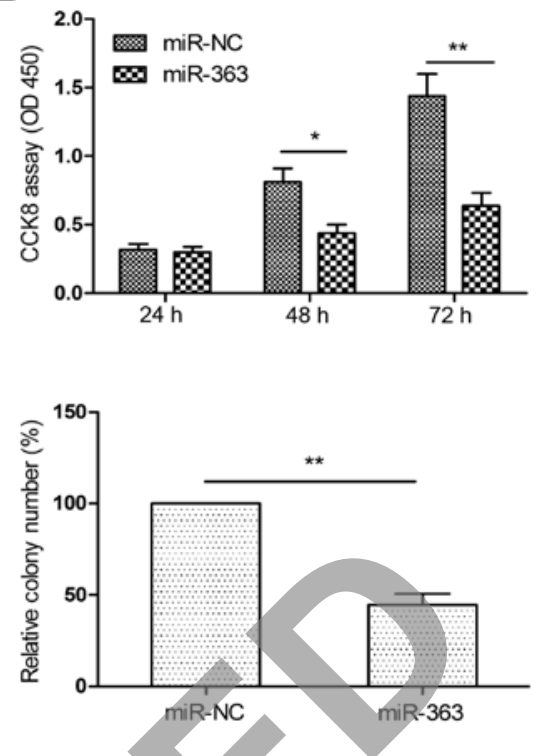

E

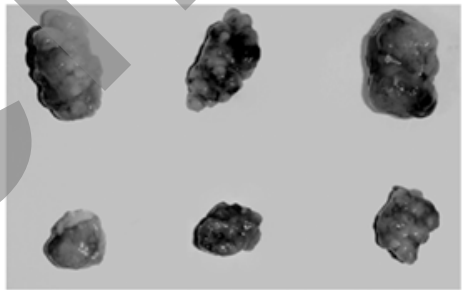

Figure 2. miR-363 inhibits HCC growth in vitro and in vivo. (A) The expression of miR-363 was assessed in HepG2 cells transfected with the miR-363 mimic or miR-NC. (B) Cell proliferation and (C) colony formation were determined in HepG2 cells transfected with miR-363 mimic or miR-NC. (D) Tumor growth curve of each group. (E.) Image of tumor tissues of each group; ${ }^{*} \mathrm{P}<0.05,{ }^{* *} \mathrm{P}<0.01$.

invasion in HCC cells using wound healing and Transwell invasion assays, respectively. Notably, we found that miR-363 overexpression significantly inhibited the migration and invasion of HepG 2 cells compared to these capacities in the miR-NC group (Fig. 3A and B). Furthermore, epithelialmesenchymal transition (EMT) has been reported to play a key role in the metastasis of HCC cells (18). To determine whether miR-363 affects molecular changes typical of EMT in HCC cells, the expression of mesenchymal markers, including $\mathrm{N}$-cadherin and vimentin and the epithelial marker, E-cadherin, was determined in the HepG2 cells. As shown in Fig. 3C, overexpression of miR-363 resulted in increased E-cadherin expression, and decreased N-cadherin and vimentin expression. These finding revealed that miR-363 inhibits HCC metastasis by regulating EMT.

E2F3 is a candidate target of miR-363 in HCC cells. To understand the mechanisms by which miR-363 suppresses HCC growth and invasion, we searched for candidate targets of miR-363 using 3 bioinformatic databases (TargetScan, miRanda and PicTar). We identified the 3'UTR of E2F3 that was able to bind to the 'seed region' of miR-363 (Fig. 4A). To confirm that miR-363 may bind to the 3'UTR of E2F3, a human E2F3 3'UTR fragment containing the binding sites of miR-363 (Fig. 4A), or the mutant sites were amplified and inserted into the psiCHECK2 vector, and miR-363 mimic or miR-NC were co-transfected into HepG2 cells and cultured for $48 \mathrm{~h}$, and then luciferase activities were measured. As predicted, miR-363 was bound to E2F3 3'UTR, resulting in markedly reduced luciferase activities (Fig. 4B). This effect was specific as miR-363 failed to suppress the luciferase activity when the binding site within E2F3 3'UTR was mutated (Fig. 4B). The results indicated that E2F3 is a downstream target of miR-363. Moreover, qRT-PCR and western blot analysis further demonstrated that overexpression of miR-363 markedly suppressed E2F3 expression at the mRNA and protein levels (Fig. 4C and D) in HepG2 cells. In addition, the E2F3 protein level in tumor tissues isolated from nude mice was determined by western blotting. The expression level of E2F3 protein in the tumor tissues with miR-363 overexpression was downregulated compared with the miR-NC group (Fig. 4D). Taken together, these data indicate that E2F3 is a direct target of miR-363 in HCC cells.

Overexpression of E2F3 reverses the tumor-suppressive effect of miR-363 in HCC. We further studied whether E2F3 is involved in the function of miR-363 in HCC cells. HepG2 cells with high expression of miR-363 were transfected with the E2F3 overexpression plasmid, and the expression of E2F3 was examined by qRT-PCR and western blotting. As shown in Fig. 5A and B, 

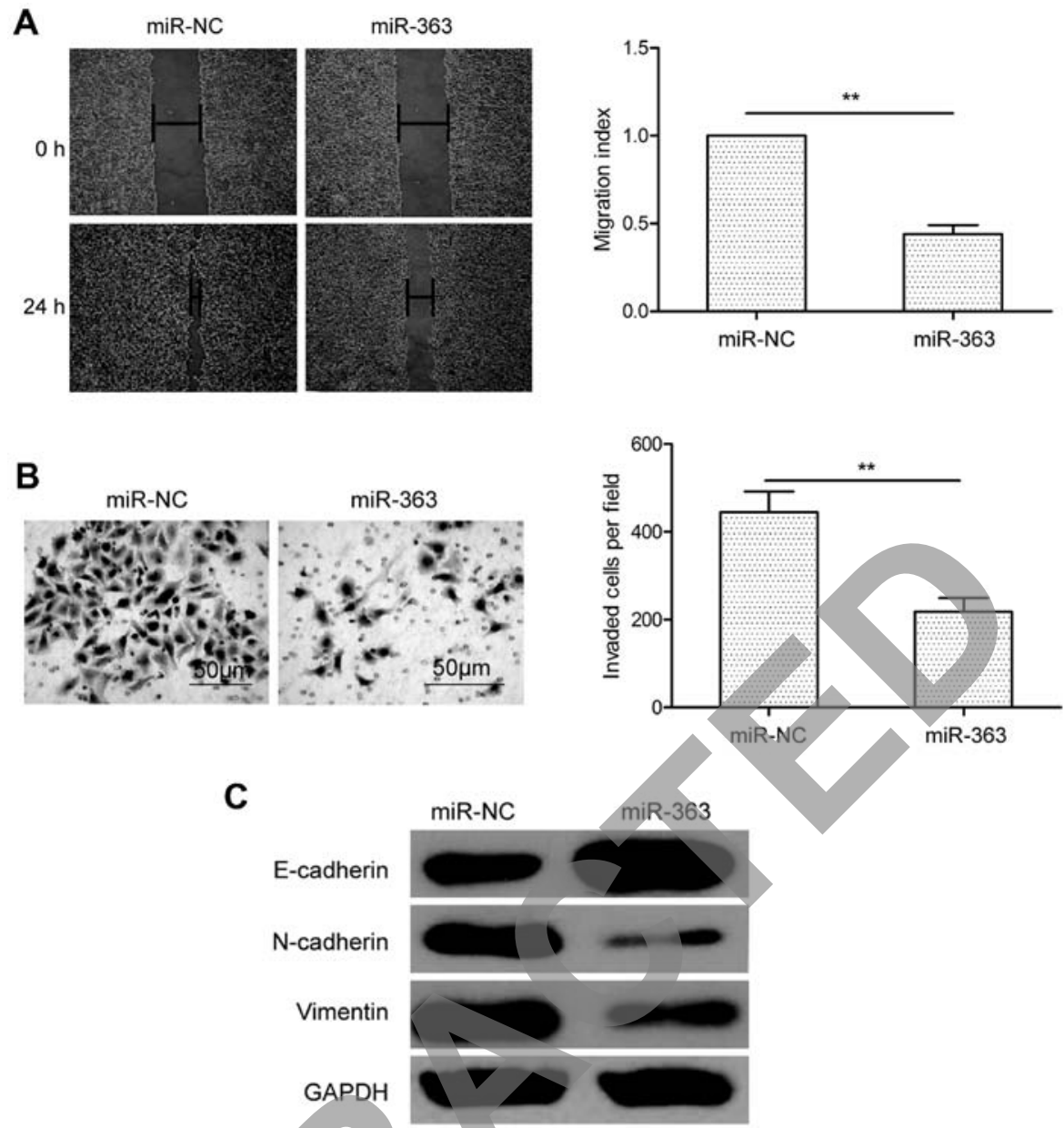

Figure 3. miR-363 inhibits HCC cell migration and invasion. (A) Cell migration ability was determined in HepG2 cells transfected with miR-363 mimic or miR-NC by wound healing assay. (B) Cell invasion ability was determined in HepG2 cells transfected with miR-363 mimic or miR-NC by Transwell invasion assay. (C) E-cadherin, $\mathrm{N}$-cadherin and vimentin protein expression were measured in HepG2 cells transfected with miR-363 mimic or miR-NC by western blotting. GAPDH was used as a control; ${ }^{* *} \mathrm{P}<0.01$.

A
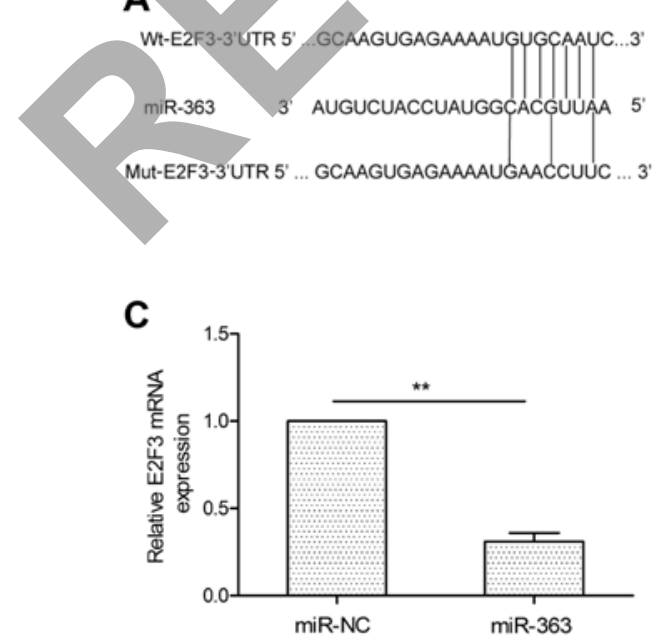

B
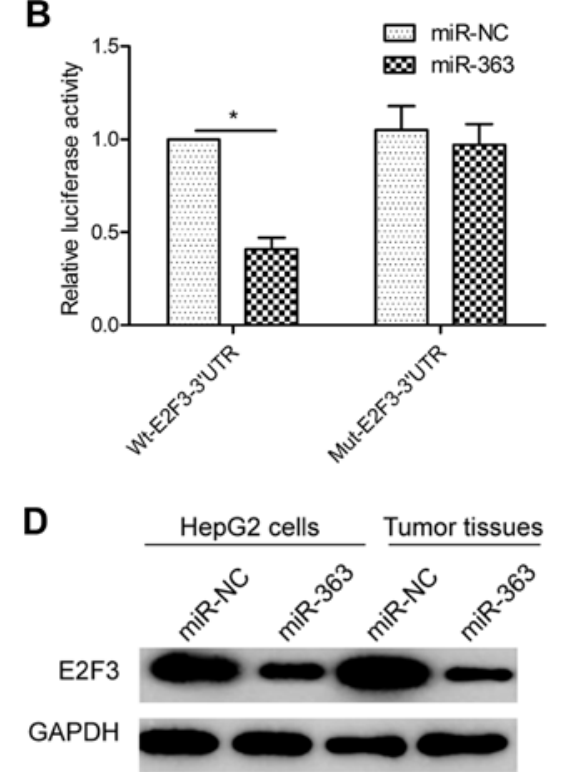

Figure 4. E2F3 is a candidate target of miR-363 in HCC cells. (A) Schematic construction of wild-type (WT) and mutant (Mut) 3'UTR of E2F3 according to miR-363 and its putative binding sequence in the 3'UTR of E2F3 (position 272-278). (B) HepG2 cells were cotransfected with wild-type or mutant 3'UTR of the E2F3 reporter plasmid and miR-363 mimic or miR-NC. The luciferase activities were determined $48 \mathrm{~h}$ after transfection. (C) The E2F3 mRNA expression level was determined in HepG2 cells transfected with miR-363 mimic or miR-NC by qRT-PCR. (D) The E2F3 protein expression level was determined in HepG2 cells transfected with miR-363 mimic or miR-NC and tumor tissues isolated form nude mouse by western blotting. GAPDH was used as a control; ${ }^{*} \mathrm{P}<0.05,{ }^{* *} \mathrm{P}<0.01$. 
A

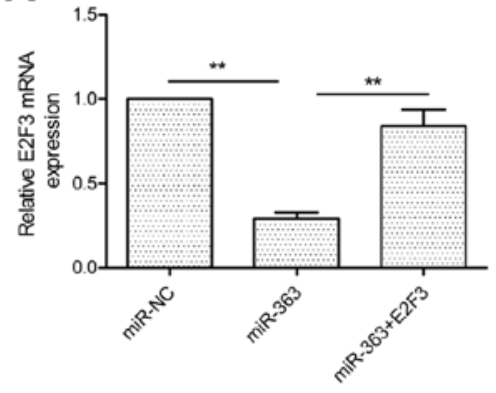

D

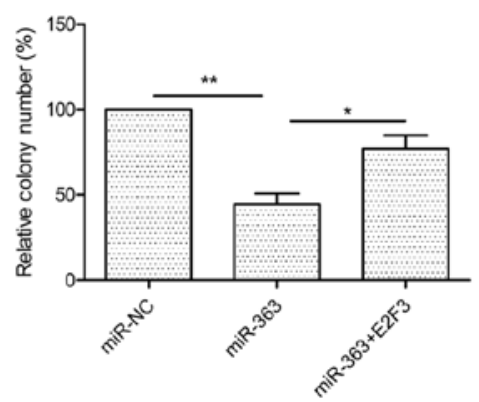

B

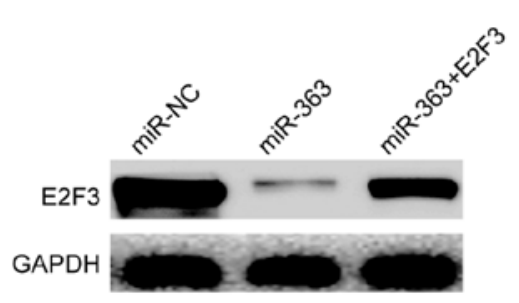

E

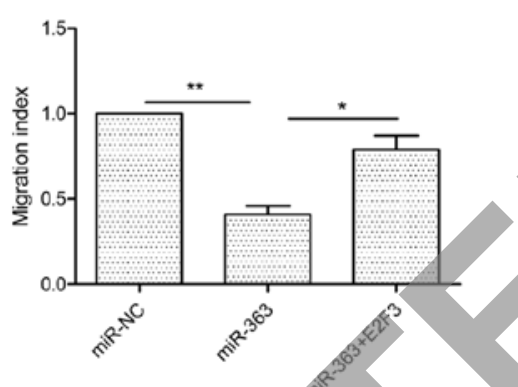

C

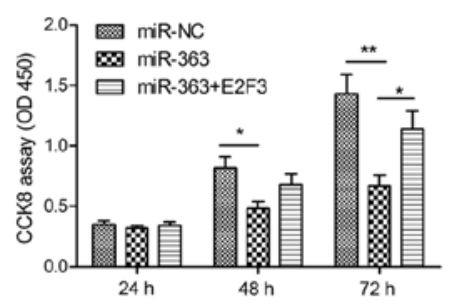

$\mathbf{F}$

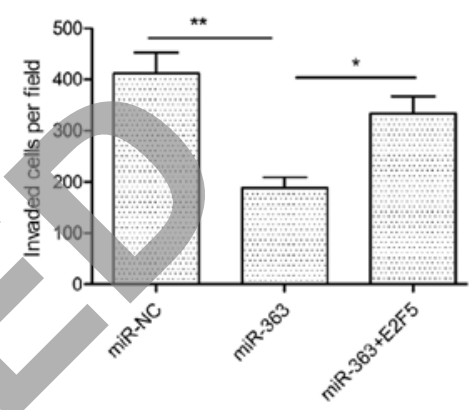

Figure 5. Overexpression of E2F3 reverses the tumor-suppressive effects of miR-363 in HCC. E2F3 expression at the (A) mRNA and (B) protein levels was determined in HepG2 cells transfected with miR-363 with/without the E2F3 oyerexpression plasmid. GAPDH was used as the internal control. (C-F) Cell proliferation, colony formation, migration and invasion were determined in HepG2 cells transfected with miR-363 with/without the E2F3 overexpression plasmid; $\mathrm{P}<0.05,{ }^{* *} \mathrm{P}<0.01$.

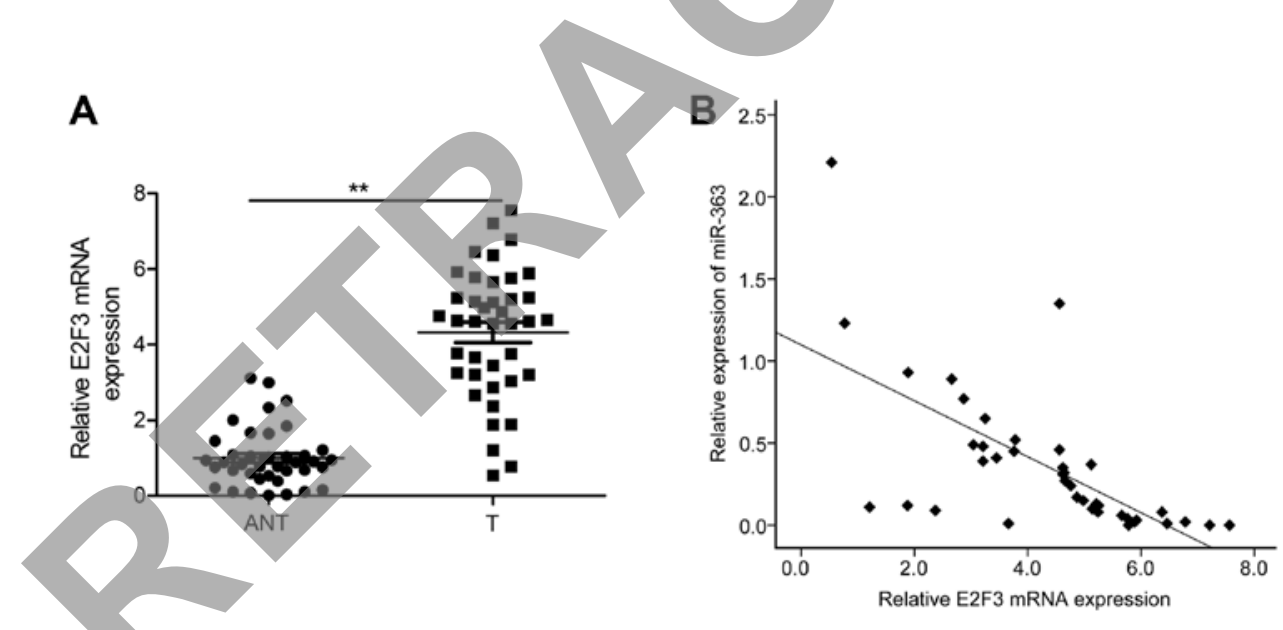

Figure 6. Inverse correlation between E2F3 and miR-363 expression in HCC tissues. (A) E2F3 mRNA expression in 40 paired HCC and adjacent non-tumor tissues was determined by qRT-PCR. GAPDH was used as an internal control. ${ }^{* *} \mathrm{P}<0.01$. ANT, adjacent non-tumor tissues. (B) The inverse correlation between miR-363 and E2F3 in 40 clinical HCC samples was determined by Pearson's correlation coefficient $(r=-0.648 ; \mathrm{P}<0.001)$.

E2F3 expression at the mRNA and protein levels was restored in the HepG2 cells. Then, we carried out CCK-8, colony formation, would healing and Transwell assays to evaluate the effect of E2F3 overexpression on cell proliferation, colony formation, migration and invasion in the HepG2 cells transfected with miR-363 with/without the E2F3 overexpression plasmid. As expected, E2F3 overexpression reversed the suppressive effect on cell proliferation, colony formation, migration and invasion in HepG2 cells induced by miR-363 overexpression (Fig. 5C-F). Therefore, our data clearly demonstrated that miR-363 inhibits $\mathrm{HCC}$ cell growth and invasion, at least in part, by targeting E2F3.

Inverse correlation between E2F3 and miR-363 expression in HCC patients. We finally examined the E2F3 mRNA expression in 40 pairs of HCC tumors and the corresponding adjacent non-tumor tissues by qRT-PCR. As shown in Fig. 6A, E2F5 mRNA expression was significantly increased in the HCC tissues compared to that noted in the adjacent nontumor tissues. Pearson's correlation analysis revealed that the expression of miR-363 was inversely correlated with the E2F3 mRNA level in the HCC tissues ( $r=-0.648 ; \mathrm{P}<0.001$; Fig. 6B).

\section{Discussion}

Emerging evidence suggests that a wide range of miRNAs play crucial roles in the development and progression of HCC, and they act as oncogenes or tumor suppressors in HCC $(9,10)$. For example, miR-449a was found to function 
as a tumor-suppressor miRNA by inhibiting cell proliferation, colony formation, migration and invasion of $\mathrm{HCC}$ by repressing ADAM10 expression (20). miR-138 inhibited proliferation, colony formation, migration and invasion in HCC cells by targeting SOX9 (19). miR-133a suppressed proliferation, colony formation, migration and invasion of HCC cells and caused cell cycle arrest at the G0/G1 stage and cell apoptosis in vitro, and decreases tumor size and weight in a nude mouse HepG2 xenograft model by targeting insulin-like growth factor-1 receptor (IGF-1R) (21). In the present study, we investigated the miR-363 expression in 40 paired cases of human HCC tissues and non-tumor tissues and in HCC cell lines by quantitative RT-PCR. Significantly, we found that miR-363 was decreased in the HCC tissues and cell lines. We also revealed that miR-363 suppressed HCC cell proliferation, colony formation, migration and invasion, as well as tumor growth in nude mice by targeting E2F3. These results imply that miR-363 may play a crucial role in HCC progression.

Recently, the role of miR-363 in carcinogenesis and cancer treatment has been a focus of research. Several studies have demonstrated that miR-363 expression is downregulated in multiple types of cancers, such as osteosarcoma (22), colorectal (12), head and neck (14), breast (15) and renal cancer (23), and neuroblastoma (24), suggesting that miR-363 functions as a potential tumor suppressor in these types of cancer. However, there are also contradictive studies. Chen et al demonstrated that overexpression of miR-363 in prostate cancer cells through transfection induced cell proliferation and positively regulated cell transformation property as well as promoted EMT in PC-3 cells by regulating c-myc (25). Zhang et al found that miR-363 overexpression promoted gastric cancer cell proliferation and chemoresistance by directly targeting the tumor suppressor F-box and WD repeat domain-containing 7 (FBW7) (13). In regards to HCC, the previous study showed that miR-363 inhibited cell proliferation by downregulating the expression of S1PR (16), and decreased cisplatin resistance of HCC cell, partly by targeting Mcl-1 (17). However, the detailed roles concerning metastasis and the mechanism of its impact on HCC cell growth and metastasis of miR-363 in HCC remain unclear. In the present study, we showed that miR-363 was downregulated in HCC cell lines and tissues, and was associated with lymph node metastasis and TNM stage, which was consistent with previous results (16). We also first demonstrated that miR-336 inhibited cell migration, invasion and EMT in HCC cells, as well as suppressed HCC tumor growth in vivo by targeting E2F3. These results further expand the function of miR-363 in $\mathrm{HCC}$ and suggest that miR-363 functions as a tumor suppressor in $\mathrm{HCC}$.

E2F3, located at $6 \mathrm{p} 22$, is a member of the E2F transcription factor family, which plays a key role in the regulation of cell proliferation (26). E2F3 has been reported to be involved in many biological processes, including apoptosis, DNA repair, differentiation, development and tumorigenesis (27). Increasing evidence suggests that dysregulation of $\mathrm{E} 2 \mathrm{~F} 3$ protein contributes to tumor formation by regulating cell proliferation, cell cycle, apoptosis, migration and invasion (28-30). Recently a study showed that E2F3 expression was upregulated in HCC tissues, and its expression was associated with poor prognosis (31), suggesting that E2F3 is an oncogene in HCC.
In addition, E2F3 has been reported to be regulated in HCC cells by several miRNAs, such as miR-141 (32), miR-144 (33), miR-503 (34), miR-214 (35) miR-424 (18) and miR-217 (36). In the present study, through luciferase activity, qRT-PCR combined with western blotting, we showed that E2F3 is a direct target of miR-363 in HCC cells. Moreover, E2F3 overexpression partially attenuated the tumor-suppressive effects of miR-363 overexpression in HCC cells, and the E2F3 mRNA level was negatively correlated with the miR-363 level in HCC tissues. These results suggest that miR-363 exerts its suppressive role in HCC, at least in part, by targeting E2F3.

In conclusion, the present study demonstrated that miR-363 is downregulated in HCC cell lines and tissues, and is associated with tumor differentiation, lymph node metastasis and TNM stage. In addition, miR-363 suppressed HCC cell proliferation, colony formation, migration and invasion in vitro, as well as HCC tumor growth in vivo by directly targeting and negatively regulating E2F3. This novel miR-363/E2F3 axis may provide new insight into the mechanisms underlying HCC progression. These results suggest that miR-363 may be a potential therapeutic target for the treatment of HCC.

\section{References}

1. Waly Raphael S, Yangde Z and Yuxiang C: Hepatocellular carcinoma: Focus on different aspects of management. ISRN Oncol 2012: 421673, 2012.

2. Mazzola A, Costantino A, Petta S, Bartolotta TV, Raineri M, Sacco R, Brancatelli G, Cammà C and Cabibbo G: Recurrence of hepatocellular carcinoma after liver transplantation: An update. Future Oncol 11: 2923-2936, 2015

3. Forner A, Llovet JM and Bruix J: Hepatocellular carcinoma. Lancet 379: 1245-1255, 2012.

4. Valinezhad Orang A, Safaralizadeh R and Kazemzadeh-Bavili M: Mechanisms of miRNA-mediated gene regulation from common downregulation to mRNA-specific upregulation. Int $\mathbf{J}$ Genomics 2014: 970607, 2014.

5. Bushati N and Cohen SM: microRNA functions. Annu Rev Cell Dev Biol 23: 175-205, 2007.

6. Hwang HW and Mendell JT: MicroRNAs in cell proliferation, cell death, and tumorigenesis. Br J Cancer 94: 776-780, 2006

7. Calin GA and Croce CM: MicroRNA signatures in human cancers. Nat Rev Cancer 6: 857-866, 2006.

8. Volinia S, Calin GA, Liu CG, Ambs S, Cimmino A, Petrocca F, Visone R, Iorio M, Roldo C, Ferracin M, et al: A microRNA expression signature of human solid tumors defines cancer gene targets. Proc Natl Acad Sci USA 103: 2257-2261, 2006.

9. Mao B and Wang G: MicroRNAs involved with hepatocellular carcinoma (Review). Oncol Rep 34: 2811-2820, 2015.

10. Giordano S and Columbano A: MicroRNAs: New tools for diagnosis, prognosis, and therapy in hepatocellular carcinoma? Hepatology 57: 840-847, 2013.

11. Wang SH, Zhang WJ, Wu XC, Weng MZ, Zhang MD, Cai Q, Zhou D, Wang JD and Quan ZW: The lncRNA MALAT1 functions as a competing endogenous RNA to regulate MCL-1 expression by sponging miR-363-3p in gallbladder cancer. J Cell Mol Med 20: 2299-2308, 2016.

12. Hu F, Min J, Cao X, Liu L, Ge Z, Hu J and Li X: MiR-363-3p inhibits the epithelial-to-mesenchymal transition and suppresses metastasis in colorectal cancer by targeting Sox4. Biochem Biophys Res Commun 474: 35-42, 2016.

13. Zhang PF, Sheng LL, Wang G, Tian M, Zhu LY, Zhang R, Zhang $\mathrm{J}$ and Zhu JS: miR-363 promotes proliferation and chemo-resistance of human gastric cancer via targeting of FBW7 ubiquitin ligase expression. Oncotarget 7: 35284-35292, 2016.

14. Chapman BV, Wald AI, Akhtar P, Munko AC, Xu J, Gibson SP, Grandis JR, Ferris RL and Khan SA: MicroRNA-363 targets myosin 1B to reduce cellular migration in head and neck cancer. BMC Cancer 15: 861, 2015.

15. Zhang R, Li Y, Dong X, Peng L and Nie X: MiR-363 sensitizes cisplatin-induced apoptosis targeting in Mcl-1 in breast cancer. Med Oncol 31: 347, 2014. 
16. Zhou P, Huang G, Zhao Y, Zhong D, Xu Z, Zeng Y, Zhang Y, Li S and He F: MicroRNA-363-mediated downregulation of S1PR1 suppresses the proliferation of hepatocellular carcinoma cells. Cell Signal 26: 1347-1354, 2014.

17. Ou Y, Zhai D, Wu N and Li X: Downregulation of miR-363 increases drug resistance in cisplatin-treated HepG2 by dysregulating Mcl-1. Gene 572: 116-122, 2015.

18. Yang H, Zheng W, Shuai X, Chang RM, Yu L, Fang F and Yang LY: MicroRNA-424 inhibits Akt3/E2F3 axis and tumor growth in hepatocellular carcinoma. Oncotarget 6: 27736-27750, 2015.

19. Liu Y, Zhang W, Liu K, Liu S, Ji B and Wang Y: miR-138 suppresses cell proliferation and invasion by inhibiting SOX9 in hepatocellular carcinoma. Am J Transl Res 8: 2159-2168, 2016.

20. Liu S, Liu K, Zhang W, Wang Y, Jin Z, Jia B and Liu Y: miR-449a inhibits proliferation and invasion by regulating ADAM10 in hepatocellular carcinoma. Am J Transl Res 8: 2609-2619, 2016.

21. Zhang W, Liu K, Liu S, Ji B, Wang Y and Liu Y: MicroRNA-133a functions as a tumor suppressor by targeting IGF-1R in hepatocellular carcinoma. Tumour Biol 36: 9779-9788, 2015.

22. Li X, Liu X, Fang J, Li H and Chen J: microRNA-363 plays a tumor suppressive role in osteosarcoma by directly targeting MAP2K4. Int J Clin Exp Med 8: 20157-20167, 2015

23. Li Y, Chen D, Li Y, Jin L, Liu J, Su Z, Qi Z, Shi M, Jiang Z, Ni L, et al: Oncogenic cAMP responsive element binding protein 1 is overexpressed upon loss of tumor suppressive miR-10b-5p and miR-363-3p in renal cancer. Oncol Rep 35: 1967-1978, 2016.

24. Qiao J, Lee S, Paul P, Theiss L, Tiao J, Qiao L, Kong A and Chung DH: miR-335 and miR-363 regulation of neuroblastoma tumorigenesis and metastasis. Surgery 154: 226-233, 2013.

25. Chen Y, Lu X, Wu B, Su Y, Li J and Wang H: MicroRNA 363 mediated positive regulation of c-myc translation affect prostate cancer development and progress. Neoplasma 62: 191-198, 2015.

26. Rady B, Chen Y, Vaca P, Wang Q, Wang Y, Salmon P and Oberholzer J: Overexpression of $E 2 F 3$ promotes proliferation of functional human $\beta$ cells without induction of apoptosis. Cell Cycle 12: 2691-2702, 2013
27. Ginsberg D: E2F3-a novel repressor of the $A R F / p 53$ pathway. Dev Cell 6: 742-743, 2004.

28. Gao Y, Li H, Ma X, Fan Y, Ni D, Zhang Y, Huang Q, Liu K, Li X, Wang L, et al: E2F3 upregulation promotes tumor malignancy through the transcriptional activation of HIF-2a in clear cell renal cell carcinoma. Oncotarget: Jul 13, 2016 (Epub ahead of print). doi: 10.18632/oncotarget.10568.

29. Rennhack J and Andrechek E: Conserved E2F mediated metastasis in mouse models of breast cancer and HER 2 positive patients. Oncoscience 2: 867-871, 2015.

30. Trikha P, Sharma N, Pena C, Reyes A, Pécot T, Khurshid S, Rawahneh M, Moffitt J, Stephens JA, Fernandez SA, et al: E2f3 in tumor macrophages promotes lung metastasis. Oncogene 35: 3636-3646, 2016.

31. Zeng X, Yin F, Liu X, Xu J, Xu Y, Huang J, Nan Y and Qiu X: Upregulation of E2F transcription factor 3 is associated with poor prognosis in hepatocellular carcinoma. Oncol Rep 31: 1139-1146, 2014.

32. Xue J, Niu YF, Huang J, Peng G, Wang LX, Yang YH and Li YQ: miR-141 suppresses the growth and metastasis of HCC cells by targeting E2F3. Tumour Biol 35: 12103-12107, 2014.

33. Cao T, Li H, Hu Y, Ma D and Cai X: miR-144 suppresses the proliferation and metastasis of hepatocellular carcinoma by targeting E2F3. Tumour Biol 35. 10759-10764, 2014.

34. Xiao F, Zhang W, Chen L, Chen F, Xie H, Xing C, Yu X, Ding S, Chen K, Guo H, et al: MicroRNA-503 inhibits the G1/S transition by downregulating cyclin D3 and E2F3 in hepatocellular carcinoma. J Transl Med 11: 195, 2013.

35. Yang Y, Chang S, Zhao Z, Hou NI, He K, Wang X, Gao L, Wang L, Cai D, Guo BO, et al: MicroRNA-214 suppresses the proliferation of human hepatocellular carcinoma cells by targeting E2F3. Oncol Lett 10: 3779-3784, 2015.

36. Su J, Wang Q, Liu Y and Zhong M: miR-217 inhibits invasion of hepatocellular carcinoma cells through direct suppression of E2F3. Mol Cell Biochem 392: 289-296, 2014.

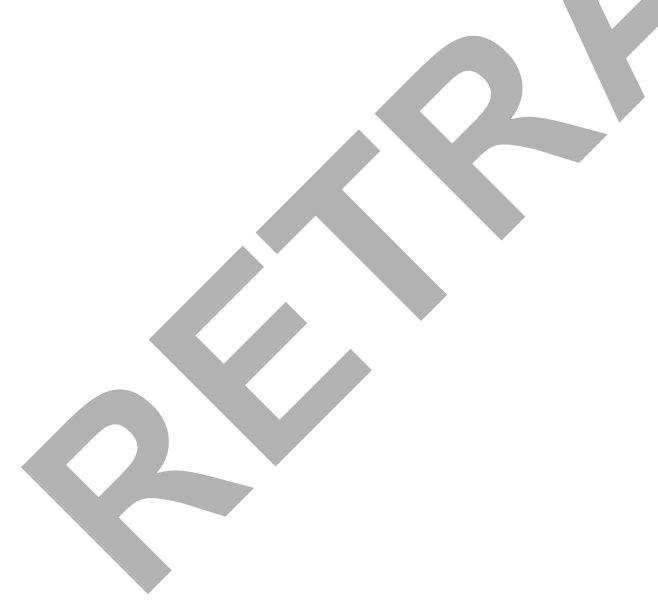

